\title{
Simple and Robust Indirect Thrust Control for Positioning of Linear Induction Motors
}

\author{
Miguel Martínez-Iturralde Non-member (University of Navarra, mmiturralde@tecnun.es) \\ Gonzalo Martínez Non-member (IDOM, gonzalo.martinez@idom.es) \\ Marcelo Castelli Non-member (University of Navarra, mcastelli@tecnun.es) \\ Andrés García Rico Non-member (University of Navarra, agarico@tecnun.es) \\ Julián Flórez Non-member (University of Navarra, florez@tecnun.es)
}

Keywords: linear induction motor, primary flux oriented control, accurate positioning

Dealing with position control of Linear Induction Motors (LIM), most strategies in bibliography are based on Secondary Flux Oriented Control (SFOC) and Direct Thrust Control (DTC). However, SFOC of linear induction motors needs complex identification methods to compensate parameter variation during operation, mainly due to local heating and end-effects. On the other hand, DTC based methods for LIMs introduce thrust ripple and have problems at low and zero speed.

In this paper, a new Indirect Thrust Control (ITC) based strategy for position control of linear induction motors that makes up for these drawbacks is presented. The primary flux control algorithm is stated for normal and full-voltage operation. Also, the position control loop design methodology and the method for automatic adjustment of compensators are presented. Experimental results are presented to evaluate the performance and sensitivity of the control strategy. Finally, some conclusions are drawn about the applicability of the new algorithm that demonstrate the main advantages versus SFOC and DTC.

There are many control strategies in bibliography that try to cope with the problem of position control of LIMs. Most of them are based on SFOC and DTC, but several problems arise.

In order to solve these problems, it was presented in [1] a primary flux oriented control that achieves smooth speed control and unsensitivity to secondary resistances variation. Afterwards, in LDIA2003, Indirect thrust control was presented [2].

In consists of a primary flux oriented control with an openprimary flux-loop that achieves very good thrust performance. Moreover, it permits the exact derivation of the thrust characteristic equation.

In this paper, this equation is used to design a position control scheme. The equation is linearised and a transfer function that accounts for the whole performance of ITC plus LIM is obtained (1).

$$
\begin{aligned}
\frac{\Delta F}{\Delta \omega_{2}}= & \frac{\frac{3 p \pi}{2 \tau} \frac{\left|\vec{\Psi}_{1}\right|^{2}}{L_{1}} \frac{1-\sigma}{\sigma}\left(\frac{r_{2}}{\sigma L_{2}}\right)^{4}}{\left(\left(\frac{r_{2}}{\sigma L_{2}}\right)^{2}+\left(\omega_{2}\right)_{o p}^{2}+\frac{\sigma \omega_{m}^{2}}{\frac{1-\sigma}{\sigma}}\right)\left(\left(\frac{r_{2}}{\sigma L_{2}}\right)^{2}+\left(\omega_{2}\right)_{o p}^{2}\right)} \\
& \frac{1}{s+\frac{r_{2}}{\sigma L_{2}}+\left(\omega_{2}\right)_{o p}} \cdots \ldots \ldots \ldots \ldots \ldots \ldots \ldots \ldots \ldots \ldots \ldots \ldots
\end{aligned}
$$

where $F$ is the thrust, $\omega_{2}$ is the slip frequency of primary flux phasor, $\omega_{m}$ is the motor electrical speed, $r_{2}$ the secondary resistance, $\sigma$ the total leakage factor, $L_{2}$ the secondary self-inductance, and the subscript $o p$ accounts for the operating point for the linearization.

The position control scheme is shown by Figure 1 .

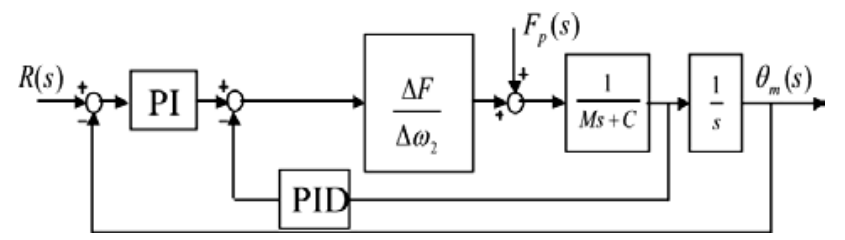

Fig. 1. Position control arrangement

$M$ and $C$ are the mass and the viscous friction coefficient. Together with this arrangement, and the transfer function (1), it is possible to obtain a global transfer function, that permits the adjustment of the compensator gains for an exact placement of the poles of the system.

Several experimental results will be presented to validate the position control strategy. A laboratory prototype has been tested with position step and ramp commands, and a complete sensitivity analysis has been made. Figure 2 shows a position step test with a $2 \mathrm{~s}$ settling time command. It is remarkable that the settling time achieved is very near to the specification.

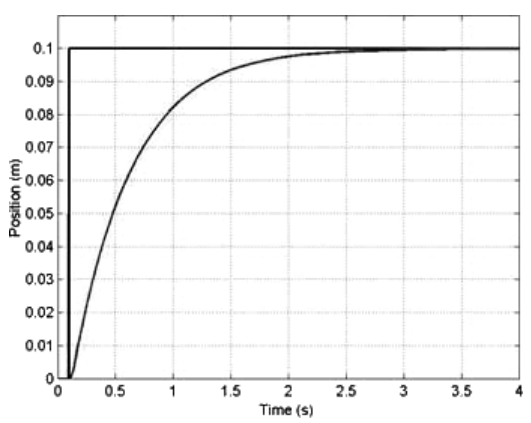

Fig. 2. Position step test

In conclusion, a new control strategy for linear induction motors is presented, capable of compensating, or even eliminating, several critic problems of the control of this kind of machines. 


\title{
Simple and Robust Indirect Thrust Control for Positioning of Linear Induction Motors
}

\author{
Miguel Martínez-Iturralde* Non-member \\ Gonzalo Martínez \\ Marcelo Castelli* \\ Non-member \\ Andrés García Rico* \\ Non-member \\ Julián Flórez* \\ Non-member \\ Non-member
}

Dealing with position control of Linear Induction Motors (LIM), most strategies in bibliography are based on Secondary Flux Oriented Control (SFOC) and Direct Thrust Control (DTC). However, SFOC of linear induction motors needs complex identification methods to compensate parameter variation during operation, mainly due to local heating and end-effects. On the other hand, DTC based methods for LIMs present thrust ripple and have problems at low and zero speeds.

In this paper, a new Indirect Thrust Control (ITC) based strategy for position control of linear induction motors that makes up for these drawbacks is presented. The position control loop design methodology and the method for automatic adjustment of compensators are described. Experimental results are presented to evaluate the performance and sensitivity of the control strategy. Finally, some conclusions are drawn about the applicability of the new algorithm that demonstrate the main advantages versus SFOC and DTC.

Keywords: linear induction motor, primary flux oriented control, accurate positioning

\section{Introduction}

It is well known that the lumped parameters of a generic induction motor are altered by temperature, magnetic saturation or skin effect, and may vary during the operation of the machine. Dealing with Linear Induction Motors, some specific effects ${ }^{(1)-(3)}$ add up more uncertainty to the real value of these parameters.

- Local heating in the conducting secondary plate modify secondary resistance value.

- The dynamic end-effects that appear at high speeds weaken the magnetic field in some areas of the airgap, and thus the magnetizing inductance value decreases.

- The motor phases have not equal electric and magnetic parameters due to the asymmetric constructive configuration and the effects mentioned above.

There are many control strategies in bibliography that try to cope with the problem of position control of LIMs. Most of them are based on SFOC and DTC, but several problems arise.

- Control strategies based on SFOC need complex methods for secondary time constant and magnetizing inductance tuning. Especially if unbalanced operation must be considered $^{(4)(5)}$.

- DTC based strategies have serious problems at low and zero speeds due to integrators used by the primary flux

\footnotetext{
* TECNUN, University of Navarra

Manuel de Lardizábal 13, 20018 San Sebastián, SPAIN

** IDOM, Advance Design and Analysis Department

Lehendakari Aguirre 3, 48014 Bilbao, SPAIN
}

estimator and present ripple in the flux and thrust responses. Moreover, the start-up performance is not optimum because there is no primary flux in absence of thrust command ${ }^{(6)}$.

In order to solve these problems, Gastli presented a primary flux oriented control ${ }^{(7)}$ that achieves smooth speed control and unsensitivity to secondary resistances variation. Afterwards, in LDIA2003, Indirect thrust control was presented ${ }^{(8)}$.

It consists of a primary flux oriented control with an open primary flux loop that achieves very good thrust performance. Moreover, it permits the exact derivation of the thrust characteristic equation.

In this paper, this equation is used to design a position control scheme. The equation is linearised and a transfer function that accounts for the whole performance of ITC plus LIM is obtained. The arrangement of the speed and position control loops is described, and, also, a very simple method for calculating the gains of the compensators is presented.

\section{Indirect Thrust Control}

ITC strategy is a primary flux oriented control with neither flux estimators, nor current compensators. Thus, it consists of a simple algorithm, very easy to implement on a DSP.

The control variables are the primary flux phasor modulus $\left|\vec{\Psi}_{1}\right|$ and the relative angular frequency of the primary flux phasor with respect to the secondary $\omega_{2}$.

In nominal conditions, the primary flux phasor modulus must be remained constant, to make the most of the motor. However, it is also possible to change this command for the flux weakening region. 
On the other hand, it is well known that, if the primary flux level is constant, the thrust $F$ is nearly proportional to the frequency $\omega_{2}$. Thus, the objective of this control strategy is to control the thrust of the motor through the variation of $\omega_{2}{ }^{(5)(6)}$. With this aim in mind, the primary voltage equation is discretized, as shown in (1) for a generic instant $k$.

$$
\vec{V}_{1}(k)=\vec{i}_{1}(k) r_{1}+\frac{\vec{\Psi}_{1}(k+1)-\vec{\Psi}_{1}(k)}{T_{s}}
$$

where:

- $\vec{V}_{1}(k)$ is the primary voltage phasor in instant $k$.

- $\vec{i}_{1}(k)$ is the primary current phasor in instant $k$.

- $\vec{\Psi}_{1}(k+1)$ and $\vec{\Psi}_{1}(k)$ are the primary flux phasors in instant $k+1$ and $k$, respectively.

- $r_{1}$ is the primary resistance and $T_{s}$ the switching period.

$\vec{\Psi}_{1}(k)$ and $\vec{i}_{1}(k)$ are known from the previous instant $k$, and from direct measurement, respectively. $r_{1}$ is measured or estimated, and $T_{s}$ is set by the inverter operation. The primary flux phasor in instant $k+1, \vec{\Psi}_{1}(k+1)$, is set by the $\left|\vec{\Psi}_{1}\right|^{*}$ command and the $\omega_{2}^{*}$ command, as shown in (2) and (3).

$$
\begin{aligned}
& \left|\vec{\Psi}_{1}(k+1)\right|=\left|\vec{\Psi}_{1}\right|^{*} \\
& \Theta_{1}(k+1)=\Theta_{1}(k)+\Delta \Theta_{m}(k)+\omega_{2}^{*} T_{s}
\end{aligned}
$$

where:

- $\Theta_{1}(k+1)$ and $\Theta_{1}(k)$ are the primary flux phasor angles in instants $k+1$ and $k$, respectively.

- $\Delta \Theta_{m}(k)$ is the variation of the electric angle of the motor in one switching period, and should be calculated as $\Theta_{m}(k+1)-\Theta_{m}(k)$. Obviously, it is not possible to measure $\Theta_{m}$ in instant $k+1$, so it is calculated from $\Theta_{m}(k)$ and $\Theta_{m}(k-1)$. This small error is corrected every switching period when considering the value of $\Theta_{1}(k)$ in (3), with the aim of not being accumulated along time.

Therefore, (1) is solved for every condition of primary flux phasor modulus and frequency $\omega_{2}$, and $\vec{V}_{1}(k)$ is obtained.

\section{Thrust Characteristic}

It is very interesting to evaluate the force response of a LIM when controlled through Indirect Thrust Control strategy. In other words, the thrust response when a $\omega_{2}$ reference and a constant value of $\left|\vec{\Psi}_{1}\right|^{*}$ is given.

This characteristic can be derived from the secondary voltage equation shown in 4 .

$$
0=r_{2} \vec{i}_{2}+\frac{d \vec{\Psi}_{2}}{d t}-j \omega_{m} \vec{\Psi}_{2}
$$

where $r_{2}$ is the secondary resistance, $\vec{i}_{2}$ is the secondary current space phasor, $\vec{\Psi}_{2}$ is the secondary flux linkage space phasor and $\omega_{m}$ is the angular motor speed.

The secondary current phasor and secondary flux phasor expressions are the following.

$$
\begin{aligned}
& \vec{i}_{2}=\frac{1}{L_{m}}\left(\vec{\Psi}_{1}-L_{1} \vec{i}_{1}\right) \ldots \\
& \vec{\Psi}_{2}=\frac{L_{2}}{L_{m}}\left(\vec{\Psi}_{1}-\sigma L_{1} \vec{i}_{1}\right) .
\end{aligned}
$$

where, $L_{m}$ is the magnetizing inductance; $L_{1}$ and $L_{2}$ are the primary and the secondary self-inductances, respectively, thus $L_{1}=l_{1}+L_{m}$ and $L_{2}=l_{2}+L_{m}$, being $l_{1}$ and $l_{2}$ the leakage inductances; and $\sigma$ is the total leakage factor. By substituting (5) and (6) into (4), the relation in (7) can be obtained.

$$
\begin{aligned}
0= & -\frac{d \overrightarrow{i_{1}}}{d t}-\frac{r_{2}}{\sigma L_{2}} \vec{i}_{1}+\frac{1}{\sigma L_{1}} \frac{d \vec{\Psi}_{1}}{d t}+\frac{r_{2}}{\sigma L_{2}} \frac{\vec{\Psi}_{1}}{L_{1}} \\
& -j \omega_{m}\left(\frac{\vec{\Psi}_{1}}{L_{1}}-\vec{i}_{1}\right) \ldots \ldots \ldots \ldots \ldots \ldots
\end{aligned}
$$

Under the condition of constant amplitude of $\vec{\Psi}_{1}$, and thus, introducing the expression $\vec{\Psi}_{1}=\left|\vec{\Psi}_{1}\right| e^{j \omega_{1} t}$ in (7), with $\omega_{1}=\omega_{m}+\omega_{2}$, it is not very difficult to solve this differential equation and, therefore to obtain the expression of $\vec{i}_{1}$. This result, the primary current phasor, can be inserted in (8),

$$
F=\frac{3 p \pi}{2 \tau}\left(\vec{\Psi}_{1} \times \vec{i}_{1}\right)
$$

resulting the expression of the thrust characteristic (9).

$$
\begin{aligned}
F= & \frac{3 p \pi}{2 \tau} \frac{\left|\vec{\Psi}_{1}\right|^{2}}{L_{1}} \frac{\left(\frac{r_{2}}{\sigma L_{2}}\right)^{2}+\left(\omega_{m}^{2}+\omega_{1}^{2}\right)}{\left(\left(\frac{r_{2}}{\sigma L_{2}}\right)^{2}+\omega_{m}^{2}+\omega_{1}^{2}\right)^{2}} \\
& {\left[\frac { r _ { 2 } } { \sigma L _ { 2 } } \omega _ { 2 } \frac { 1 - \sigma } { \sigma } \left(1-\cos \left(\omega_{2} t\right) e^{\left.-\frac{r_{2}}{\sigma L_{2}} t\right)+}\right.\right.} \\
& \left.\left(\left(\frac{r_{2}}{\sigma L_{2}}\right)^{2} \frac{\omega_{2}^{2}}{\sigma}\right) \sin \left(\omega_{2} t\right) e^{-\frac{r_{2}}{\sigma L_{2}} t}\right]+ \\
& \frac{3 p \pi}{2 \tau}\left|\vec{\Psi}_{1}\right|\left(i_{1 d 0} \sin \left(\omega_{2} t\right)-i_{1 q 0} \cos \left(\omega_{2} t\right)\right) e^{-\frac{r_{2}}{\sigma L_{2}} t}
\end{aligned}
$$

where $\tau$ is the pole pitch, $p$ is the number of pole pairs, and $i_{d 0}$ and $i_{q 0}$ are the initial conditions of the primary current in the static $d-q$ reference frame. This expression was first presented by Takahashi and Noguchi ${ }^{(6)}$ for the particular case of zero motor speed.

The transient terms of (9) quickly decay to zero, as the value of $\frac{r_{2}}{\sigma L_{2}}$ is very high in linear induction motors. The main reason is that this value specially depends on the secondary resistance value, which is high in conducting secondary plate LIMs, and on the secondary leakage inductance, which has a very low value ${ }^{(3)}$. However, a very fast, but not negligible, transient in the thrust response is not desirable. Therefore, a proper design of the position control loop is essential in order to minimise this transient term.

\section{Position Control}

4.1 Transfer Function Expression (9) was used to derive a transfer function that takes into account the dynamic performance of a LIM controlled by ITC strategy. This transfer function was later used to design the position control loops. For this purpose, (9) was linearised around a generic operating point and the Laplace transformation was applied to the resultant expression. The transfer function obtained is a quite complex fourth order function ${ }^{(9)}$, but which is very easily reduced to a first order transfer function with practically no loss of information (10).

$$
\frac{\Delta F}{\Delta \omega_{2}}=\frac{K}{s+\frac{r_{2}}{\sigma L_{2}}+\left(\omega_{2}\right)_{o p}}
$$


where $\left(\omega_{2}\right)_{o p}$ is the slip frequency of the operating point used for the linearization and $K$ is calculated by (11).

$$
K=\frac{\frac{3 p \pi}{2 \tau} \frac{\left|\vec{\Psi}_{1}\right|^{2}}{L_{1}} \frac{1-\sigma}{\sigma}\left(\frac{r_{2}}{\sigma L_{2}}\right)^{4}}{\left(\left(\frac{r_{2}}{\sigma L_{2}}\right)^{2}+\left(\omega_{2}\right)_{o p}^{2}+\frac{\sigma \omega_{m}^{2}}{1-\sigma}\right)\left(\left(\frac{r_{2}}{\sigma L_{2}}\right)^{2}+\left(\omega_{2}\right)_{o p}^{2}\right)}
$$

In order to demonstrate the validity of this aproximation, Fig. 1 presents the thrust responses to a $\omega_{2}$ step input using (9) for several motor speeds and operating points, and the responses to the same inputs using the simplified transfer function in (10). The parameters of the linear induction motor used for this comparison are shown in Table I. The simplified transfer function has little discrepancy from the exact calculation of the thrust. Therefore, it can be used to design the speed and position control loops.

4.2 Position Control Loops The arrangement for position control of a LIM that uses ITC as thrust and flux control algorithm is shown in Fig. 2. It consists of a PID feedback compensator in the speed loop together with a PI cascade compensator in the position loop. In Fig. $1, R_{x}(s)$ and $R_{v}(s)$ are the position and speed references, respectively, $F_{p}(s)$ is the disturbance force, and $M$ and $C$ are the mass and the viscous damping coefficient of the system, respectively.

This arrangement is the only one that provides a close loop system that presents neither steady state error with position step commands, nor with disturbance force step inputs.

In order to adjust the compensator gains for a required system dynamics a very simple method based on pole assignment has been developed.

4.3 Pole Assignment The characteristic equation of the close loop system is shown in (11).
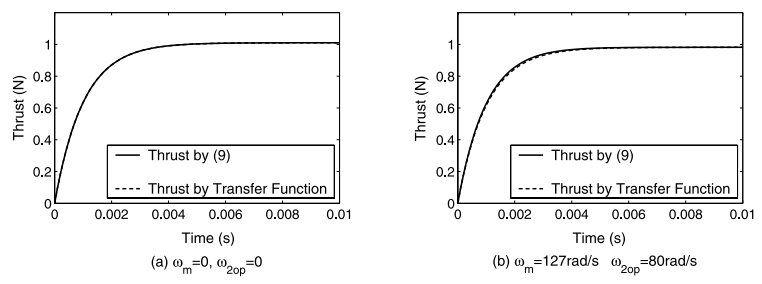
Time (s) (b) $\omega_{\mathrm{m}}=127 \mathrm{rad} / \mathrm{s} \quad \omega_{20 \mathrm{p}}=80 \mathrm{rad} / \mathrm{s}$
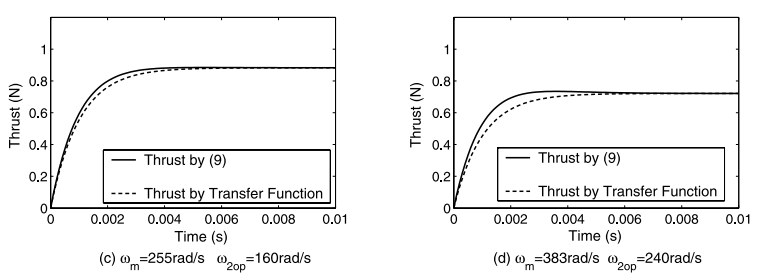

Fig. 1. Transfer function aproximation

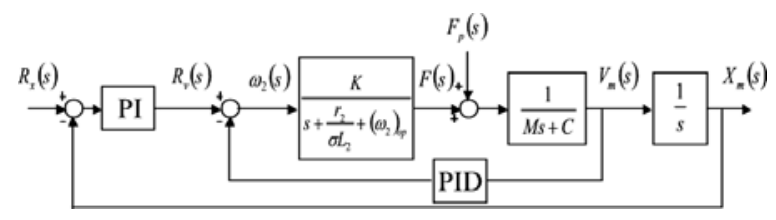

Fig. 2. Position control scheme

$$
\begin{aligned}
s^{4} & +\left(\frac{r_{2}}{\sigma L_{2}}+\left(\omega_{2}\right)_{o p}+\frac{C}{M}+\frac{K}{M} K_{v d}\right) s^{3} \\
& +\left(\left(\frac{r_{2}}{\sigma L_{2}}+\left(\omega_{2}\right)_{o p}\right) \frac{C}{M}+\frac{K}{M} K_{v p}\right) s^{2} \\
& +\frac{K}{M}\left(K_{v i}+K_{d p}\right) s+\frac{K}{M} K_{d i}=0 \ldots
\end{aligned}
$$

- $K_{d p}$ and $K_{d i}$ are the proportional and integral gains of the PI compensator.

- $K_{v p}, K_{v i}$ and $K_{v d}$ are the proportional, integral and derivative gains of the PID compensator.

It is possible to identify every term of equation (11) with the terms of a desired characteristic equation that consists of two electrical poles and two mechanical poles (12).

$$
\left(s^{2}+2 \xi_{e l} \omega_{e l} s+\omega_{e l}^{2}\right)\left(s^{2}+2 \xi_{m e c} \omega_{m e c} s+\omega_{m e c}^{2}\right)=0
$$

- $\xi_{e l}$ and $\omega_{e l}$ are the damping and frequency of the desired electrical poles and,

- $\xi_{m e c}$ and $\omega_{m e c}$ are the damping and frequency of the desired mechanical poles.

This way, the compensators gains are calculated by the following expressions:

$$
\begin{aligned}
& K_{v d}=\frac{M}{K}\left(2 \xi_{e l} \omega_{e l}+2 \xi_{m e c} \omega_{m e c}\right. \\
& \left.-\frac{r_{2}}{\sigma L_{2}}-\left(\omega_{2}\right)_{o p}-\frac{C}{M}\right) \\
& K_{v p}=\frac{M}{K}\left(\omega_{m e c}^{2}+\omega_{e l}^{2}+2 \xi_{e l} \omega_{e l} 2 \xi_{m e c} \omega_{m e c}\right. \\
& \left.-\left(\frac{r_{2}}{\sigma L_{2}}+\left(\omega_{2}\right)_{o p}\right) \frac{C}{M}\right) \\
& K_{v i}+K_{d p}=\frac{M}{K}\left(2 \xi_{e l} \omega_{e l} \omega_{m e c}^{2}+2 \xi_{m e c} \omega_{m e c} \omega_{e l}^{2}\right) \\
& K_{d i}=\frac{M}{K} \omega_{e l}^{2} \omega_{m e c}^{2}
\end{aligned}
$$

These gains are subjected to variation with the motor speed, and also depend on the operating point value of the slip frequency $\left(\omega_{2}\right)_{o p}$. Therefore, it is convenient to establish some criteria to adjust the compensators in case of a wide range of speed and thrust developed by the motor. A common sense solution is to define several ranges of speed and thrust where different calculated gains would act. For example, three regions of speed: low speed, medium-nominal speed and high speed (that would coincide with flux-weakening region), and another three regions of thrust: no-load thrust, nominal thrust and maximum thrust. This solution would result in a look-up table whith nine different values of the gains depending on the operation of the motor. The number of ranges adopted, and the sensitivity of the drive performance to a sudden change in the compensator gains values, depend on the characteristics of the motor, specially on the value of $\frac{r_{2}}{\sigma L_{2}}$. In the future, further research will be made in this direction.

\section{Experimental Results}

In this section, some experimental results are presented 
that validate the position control strategy. For this purpose, the algorithm was programmed and applied to the prototype shown in Fig. 3. This prototype is a $1 \mathrm{~kW}$ single sided linear induction motor designed and constructed in TECNUN ${ }^{(10)}$. The lumped parameters of the LIM prototype were obtained by applying an identification method that combines standstill and no-load tests ${ }^{(10)}$. Their values are shown in Table 1. where $l_{1}$ and $l_{2}$ are the leakage inductances.

Fig. 4 shows the motor response to a position step command. The adjustment of the compensators gains is made for $\omega_{m e c}=2 \frac{\mathrm{rad}}{\mathrm{s}}$ and $\xi_{m e c}=1$, so that the theoretical settling time is $t_{s}=2 \mathrm{~s}$, and $\omega_{e l}=500 \frac{\mathrm{rad}}{\mathrm{s}}$ and $\xi_{e l}=1$, so that the electrical poles do not disturb the mechanical response. This adjustment has been chosen in order to not to saturate the power supply in the starting-up of the motor, situation that is very common in this low-power laboratory prototype. The compensator gains have been calculated using equations (14) to (17) for $\omega_{m}=0$ and $\left(\omega_{2}\right)_{o p}=0$, just because speed and thrust requirements are low. It is remarkable the good performance achieved by the system, that presents an experimental settling time of exactly $2 \mathrm{~s}$.

5.1 Sensitivity Some tests were made to evaluate the

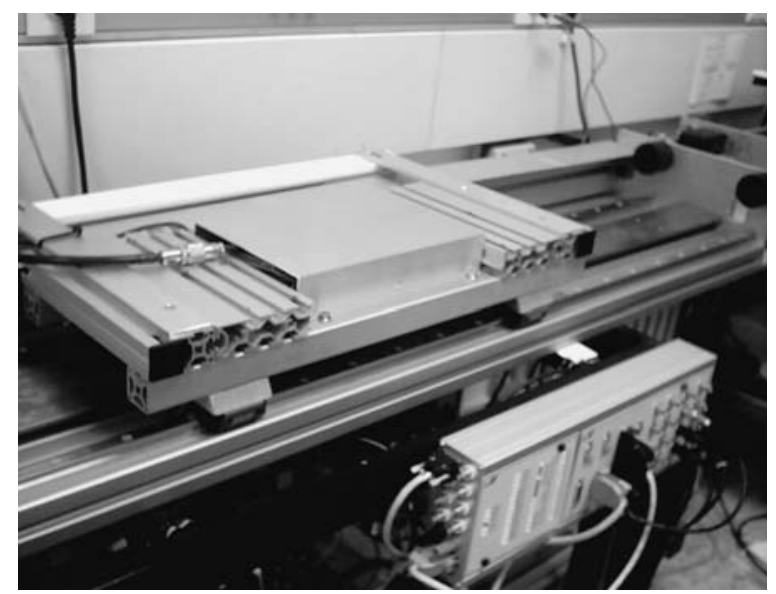

Fig. 3. Linear induction motor prototype

Table 1. LIM lumped parameters and dimensions

\begin{tabular}{l|l|l|l|l}
\hline$r_{1}$ & $r_{2}$ & $l_{1}$ & $L_{m}$ & $l_{2}$ \\
\hline \hline $52,8 \Omega$ & $44,8 \Omega$ & $0,137 \mathrm{H}$ & $0.295 \mathrm{H}$ & $0,004 \mathrm{H}$ \\
\hline \hline$p$ & $\tau$ & prim. width & sec. height & prim. weight \\
\hline \hline 4 & $50 \mathrm{~mm}$ & $50 \mathrm{~mm}$ & $4 \mathrm{~mm}$ & $10 \mathrm{~kg}$ \\
\hline \hline
\end{tabular}

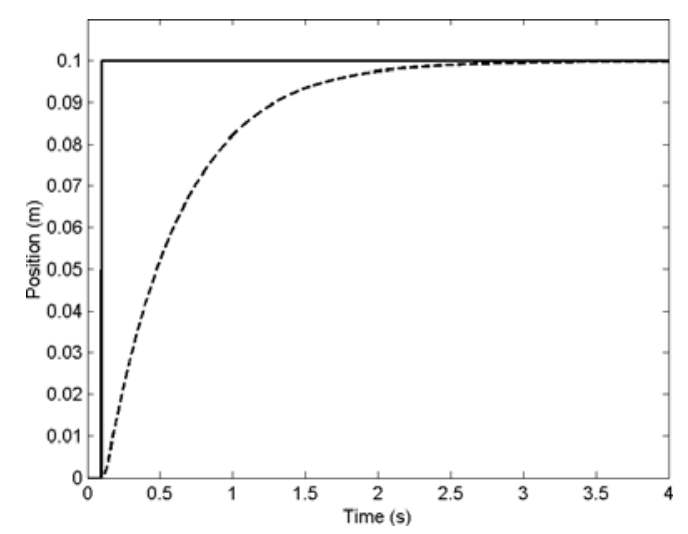

Fig. 4. ITC Position step response sensitivity of the control to the variation of the motor parameters, and to compare the ITC position control performance with SFOC and DTC performance.

Fig. 5 and Fig. 6 show various responses of the proposed control and SFOC, respectively, to a $0.1 \mathrm{~m}$ step command. The gains of the compensators are calculated for the same specifications as Fig. 3 test, but with three different cases of precision in the estimation of the secondary resistance value.

- case 1: every LIM parameter is estimated correctly.

- case 2: the estimated secondary resistance value is double of the real value.

- case 3: the estimated secondary resistance value is half of the real value.

The performance of ITC based position control strategy varies with the incorrect estimation of the secondary resistance value because this estimation is used to calculate the compensator gains. On the other hand, although the SFOC uses this value to estimate the secondary flux phasor angle its position performance vary very little from case 1 .

With this results, SFOC seems to be more robust to the incorrect estimation of the secondary resistance. However, attending to the energy consumption of the tests (Table 2), it can be seen clearly that a variation of the value of $r_{2}$ causes an

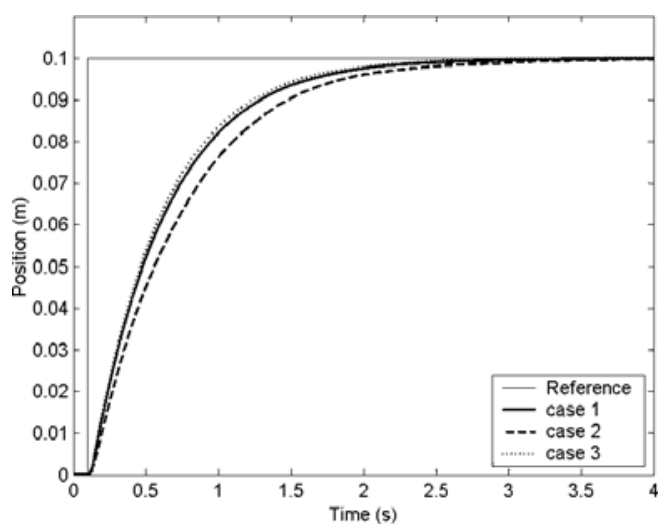

Fig. 5. ITC position step response for different estimations of $r_{2}$

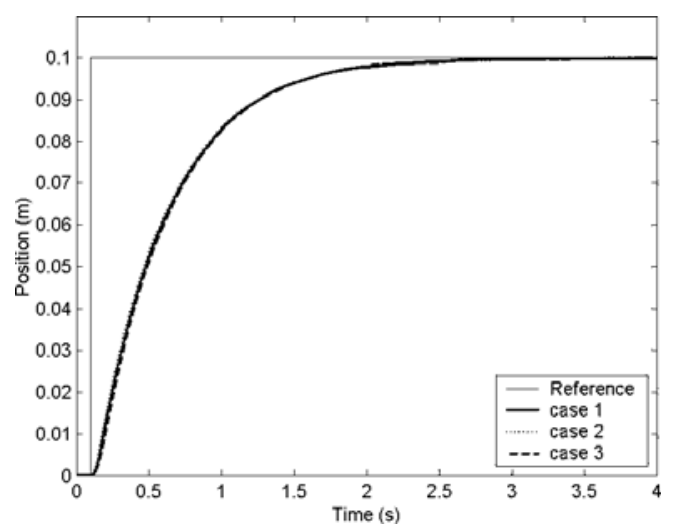

Fig. 6. SFOC position step response for different estimations of $r_{2}$

Table 2. Energy values in sensitivity to $r_{2}$ tests

\begin{tabular}{l|l|l|l}
\hline & Case 1 & Case 2 & Case 3 \\
\hline \hline ITC & $1009,8 \mathrm{~J}$ & $968,8 \mathrm{~J}$ & $1036,6 \mathrm{~J}$ \\
SFOC & $978,6 \mathrm{~J}$ & $874,8 \mathrm{~J}$ & $1240,3 \mathrm{~J}$ \\
\hline
\end{tabular}




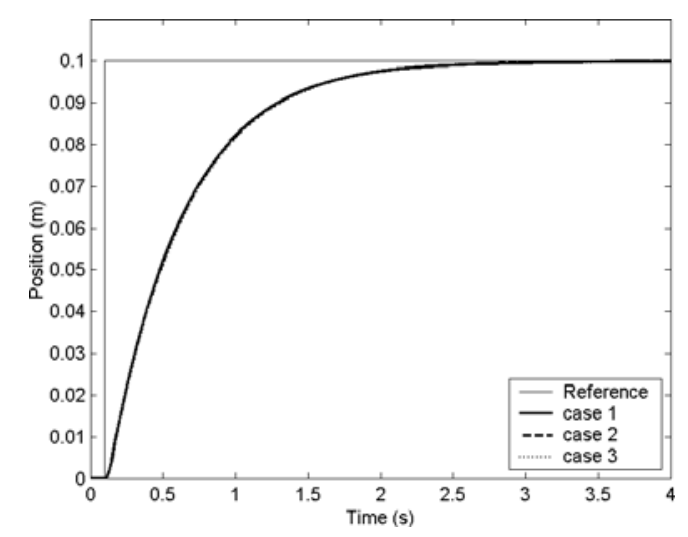

Fig. 7. ITC position step response for different estimations of $r_{1}$

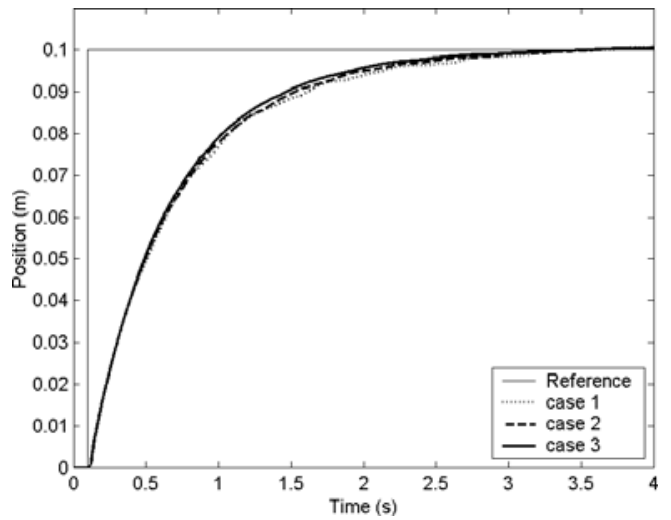

Fig. 8. DTC position step response for different estimations of $r_{1}$

important variation in the energy consumed during the test. The energy value of the test of ITC with $r_{2}$ correctly estimated is a little higher than the energy value of SFOC in optimal conditions. This is because ITC is a primary flux control, and does not maintain exactly 90 electrical between currents and secondary flux, as SFOC does. However, in the other two cases, while the energy consumption values of the ITC based strategy vary $-4 \%$ and $+2,6 \%$ from the case 1 value, the SFOC energy values vary $-10,6 \%$ and $26,7 \%$ from the case 1 value.

This is because the real secondary flux level has decreased or increased in the motor while $r_{2}$ estimated value is higher or lower than the real value. On the other hand, there is no variation in the primary flux level when ITC control strategy is used, as it does not depend on the $r_{2}$ value. For that reason, the energy variation is only caused by the errors in the compensator gains, and results to be very low.

The main advantage of the ITC based position control strategy versus SFOC, is that the uncertainty of the $r_{2}$ value is located in the outer loops (speed and position loops), instead of in the flux estimator. Therefore, the energy provided to the motor is maintained, demostrating that this strategy is more robust to this uncertainty. Another advantage is that it is easier to implement any kind of adaptive system to correct the compensator gains than to correct the flux estimator.

A very similar test has been made to evaluate the sensitivity of the control strategy to the variation of the primary resistance, and also to compare it to DTC strategy. The po-
Table 3. Energy values in sensitivity to $r_{1}$ tests

\begin{tabular}{l|l|l|l}
\hline & Case 1 & Case 2 & Case 3 \\
\hline \hline ITC & $1009,8 \mathrm{~J}$ & $983,1 \mathrm{~J}$ & $955 \mathrm{~J}$ \\
DTC & $1050 \mathrm{~J}$ & $576 \mathrm{~J}$ & $422 \mathrm{~J}$ \\
\hline
\end{tabular}

sition tests are exactly the same as previous tests, but three different cases have been performed:

- case 1: every LIM parameter is estimated correctly.

- case 2: the estimated primary resistance value is $80 \%$ of the real value.

- case 3: the estimated primary resistance value is $50 \%$ of the real value.

It is not possible to make tests with a estimated primary resistance value higher than real value because this will surely lead to unstability. Fig. 7 shows the performance of ITC based strategy for the three cases, and Fig. 8 shows the performance of the same three cases for DTC.

It is remarkable the good performance of both strategies to a variation of the primary resistance. However, the proposed control strategy presents smoother and closer responses. On the other hand, attending to the energy consumption values for the three cases, presented in Table 3, the ITC strategy is clearly more robust than DTC to the variation of $r_{1}$. It presents an energy variation of $-2,7 \%$ and $-5,2 \%$ from the value of case 1 , while DTC presents an energy variation of $-45 \%$ and $-59,8 \%$ from the case 1 value, as the primary flux decreases abnormally in the motor.

\section{Conclusions}

In this paper, a new control strategy for positioning of linear induction motors is presented. It is based on the indirect thrust control strategy, and its main advantages derive from the simplicity of the system model that is obtained. The desired motor dynamics can be automatically established through the calculation of the gains of the compensators.

The experimental results presented demonstrate its good performance and its main advantages versus SFOC and DTC, that, to sum up, are the following:

On one hand, in ITC strategy the uncertainty about the $r_{2}$ real value is located in the outer loops, while in SFOC strategy is located in the secondary flux estimator. Therefore, there is no variation in the flux of the motor even when an incorrect value is used, and it is easier to correct this deviation by any kind of adaptive system.

On the other hand, the proposed strategy presents better results than DTC strategy attending to positioning performance, energy consumption and unsensitivity to $r_{1}$ variation.

\section{Acknowledgment}

The authors wish to express their gratitude to the Ministerio de Educación y Ciencia of Spain for the financial support referenced DPI2004-02298, and to the Basque Government, for the financial support referenced T310103.

(Manuscript received Dec. 1, 2005,

revised May 9, 2006)

\section{References}

( 1 ) E.R. Laithwaite: "Induction Machines for Special Purposes", Butterworth \& Co. (Publishers) Ltd. London (1966) 
( 2 ) J.F. Gieras: Linear induction drives, Monographs in Electrical and Electronic Engineering. Oxford University Press (1994)

( 3 ) S.A. Nasar and I. Boldea: Electric linear actuators and generators, Cambridge University Press (1997)

( 4 ) B.K. Bose: Power electronics and variable frequency drives, IEEE Press, New York (1997)

( 5 ) P. Vas: Sensorless Vector and Direct Torque Control, Clarendon Press, Oxford (1998)

( 6 ) I. Takahashi and T. Noguchi: "A new quick-response and high efficiency control strategy of an induction motor", IEEE Trans. on Industry Appl., Vol.IA22, No.5 (1986-9/10)

( 7 ) A. Gastli: "Improved Field oriented Control of a LIM Having joints in its Secondary Conductors", IEEE Trans. on Energy Conversion, Vol.17, No.3, pp.349-355 (2002-9)

( 8 ) M. Martínez-Iturralde, A. García Rico, and J. Flórez: "Control strategies for linear induction motors", Proc. of the LDIA 2003 Conference, Birmingham (UK) (2003-9)

( 9 ) M. Martínez-Iturralde: "Design of a new vectorial control strategy for linear induction motors", PhD. Thesis, University of Navarra (June 2005) (in spanish)

(10) J. Atencia, A. García, J. Rico, Flórez: "A low cost linear induction motor for laboratory experiments", Int. J. Electr. Eng. Educ. (UK), Vol.38, No.2, pp.117-34 (2001-4)

Miguel Martínez-Iturralde (Non-member) received the Industrial

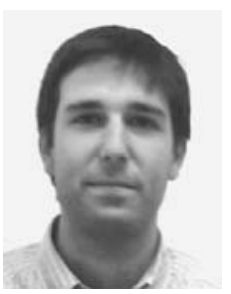
Engineering degree from Tecnun, School of Engineering of the University of Navarra in 2001, and the doctoral degree from the same university in 2005. At the moment, he is Researcher at the Electrical Machines Research Group, in Tecnun (University of Navarra). His research topics are design and control of electrical drives and levitation systems. He is IEEE member.

Gonzalo Martínez (Non-member) received the Industrial Engineer-

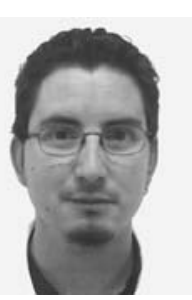
ing degree from the School of Engineering of the Universidad de Navarra, in 2000, and the doctoral degree from the same university in 2005 in the field of design and optimization of PMLSMs. He is currently working in the Advanced Design and Analysis Department of IDOM Ingeniería, Arquitectura y Consultoría, S.A., as engineer specialist in electrical machines and drives. He is IEEE member.
Marcelo Castelli (Non-member) received the Industrial Engineering

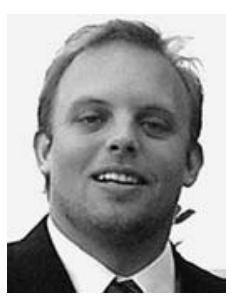
degree from the School of Engineering of the University of Montevideo, in 2004. He is currently working towards the doctoral degree at the Electrical Machines Research Group in Tecnun (University of Navarra). His research interests include the electromagnetic design of magnetic levitation systems.

Andrés García Rico (Non-member) received his Energetic Engi-

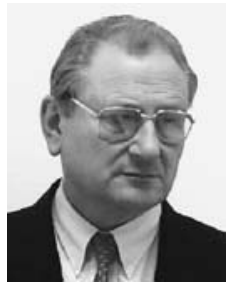
neering degree from the University of Barcelona in 1970, and the doctorate degree from the University of Navarra in 1978, in the field of Electric Machinery. He is currently Titular Professor of Electric Machinery and Industrial Projects at the School of Engineering in San Sebastian (University of Navarra). He also is Principal Researcher of the Electrical Machines Research Group, in Tecnun (University of Navarra). He holds two industrial patents and has written two books and several papers about Electrical and Power Engineering. He is IEEE member.

Julián Flórez (Non-member) received his Industrial Engineering de-

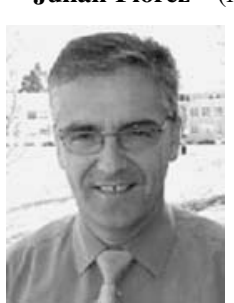
gree from the Universidad de Navarra (1980), and obtained his Ph.D. degree in the University of Manchester, Institute of Science and Technology UMIST (1985), in the field of Adaptive Control. From 1985 to 1997 he worked as Researcher in the Center of Study and Technical Research of Gipuzkoa (CEIT), where he collaborated in several research projects related to Electrical and Industrial Engineering with a marked industrial focus. Since 1985 he has been working as Titular Professor in the School of Engineering of the Universidad de Navarra. Since 2001, he is Principal Researcher and General Manager of VICOMTech, an applied research institute on Computer Graphics. He holds some industrial patents and has written more than 50 research papers in different areas of Industrial and Electrical Engineering. He is IEEE member. 